\title{
Differential Sensitivities of Plant and Animal Mitochondria to the Herbicide Paraquat
}

\author{
Joaquim A. F. Vicente, ${ }^{1}$ Francisco Peixoto, ${ }^{2}$ M. Ludovina Lopes, ${ }^{1}$ \\ and Vitor M. C. Madeira ${ }^{2}$ \\ ${ }^{1}$ Departamento de Botânica, Faculdade de Ciências e Tecnologia da Universidade de Coimbra, 3004-517 Coimbra, Portugal; \\ E-mail: jvicente@ci.uc.pt \\ ${ }^{2}$ Departamento de Zoologia, Faculdade de Ciências e Tecnologia da Universidade de Coimbra, 3004-517 Coimbra, Portugal
}

Received 29 June 2001; revised 10 September 2001; accepted 24 September 2001

\begin{abstract}
Paraquat herbicide is toxic to animals, including humans, via putative toxicity mechanisms associated to microsomal and mitochondrial redox systems. It is also believed to act in plants by generating highly reactive oxygen free radicals from electrons of photosystem I on exposure to light. Paraquat also acts on non-chlorophyllous plant tissues, where mitochondria are candidate targets, as in animal tissues. Therefore, we compared the interaction of paraquat with the mitochondrial bioenergetics of potato tuber, using rat liver mitochondria as a reference. Paraquat depressed succinate-dependent mitochondrial $\Delta \psi$, with simultaneous stimulation of state $4 \mathrm{O}_{2}$ consumption. It also induced a slow time-dependent effect for respiration of succinate, exogenous NADH, and $N, N, N^{\prime}, N^{\prime}$ tetramethyl- $p$-phenylenediamine (TMPD)/ascorbate, which was more pronounced in rat than in potato mitochondria. However, with potato tuber mitochondria, the $\Delta \psi$ promoted by complex-I-dependent respiration is insensitive to this effect, indicating a protection against paraquat radical afforded by complex I redox activity, which was just the reverse of to the findings for rat liver mitochondria. The experimental set up with the tetraphenyl phosphonium $\left(\mathrm{TPP}^{+}\right)$-electrode also indivated production of the paraquat radical in mitochondria, also suggesting its accessibility to the outside space. The different activities of protective
\end{abstract}

Correspondence to: Joaquim A. F. Vicente.

Contract Grant Sponsor: Fundação para a Ciência e a Tecnologia of Portugal.

Contract Grant Number: PBIC/C/BIO/1985/95 and Praxis 2/2.1/BIO/1156/95.

Abbreviations: BSA, bovine serum albumin; FCCP, carbonyl cyanide $p$-trifluoromethoxyphenyl-hydrazone; $\mathrm{MDH}$, malondialdehyde; Mn-SOD, manganese-superoxide dismutase; NBT, nitroblue tetrazolium; $\mathrm{O}_{2}{ }^{-}$, superoxide; $\mathrm{PQ}^{2+}$, oxidized paraquat; $\mathrm{PQ}^{+}$, paraquat radical; TMPD, $N, N, N^{\prime}, N^{\prime}$-tetramethyl- $p$-phenylenediamine; $\mathrm{TPP}^{+}$, tetraphenylphosphonium; $\Delta \psi$, transmembrane electrical potential; SOD, superoxide dismutase.

(C) 2001 John Wiley \& Sons, Inc.

DOI 10.1002/jbt.10010 antioxidant agents can contribute to explain the different sensitivities of both kinds of mitochondria. Values of SOD activity and $\alpha$-tocopherol detected in potato mitochondria were significantly higher than in rat mitochondria, which, in turn, revealed higher values of lipid peroxidation induced by paraquat. (c) 2001 John Wiley \& Sons, Inc. J Biochem Mol Toxicol 15:322-330, 2001

KEY WORDS: Paraquat; Mitochondria; Herbicide Toxicity

\section{INTRODUCTION}

Paraquat (1,1'-dimethyl-4,4'-bipyridylium dichloride), a bipyridinium herbicide widely used in agriculture, is very effective against a wide variety of weeds [1]. However, the compound is strongly toxic to plants and animals, including humans [2,3]. It is a postmergence contact herbicide, acting by accepting electrons from photosystem I (PSI) of chloroplast thylakoids [4-6].

In aqueous solution, the paraquat divalent cation $\left(\mathrm{PQ}^{2+}\right)$ accepts electrons, forming the blue paraquat radical $\left(\mathrm{PQ}^{+}\right)$, which reacts very rapidly with molecular oxygen to yield superoxide anion, $\mathrm{O}_{2}{ }^{--}$[7]. Under aerobic conditions, $\mathrm{PQ}^{+}$promotes continuous formation of $\mathrm{O}_{2}{ }^{-}$, undergoing repeated redox cycles. The $\mathrm{O}_{2}{ }^{-}$is relatively harmless but it gets converted into hydrogen peroxide $\left(2 \mathrm{H}^{+}+2 \mathrm{O}_{2}{ }^{-}<->\mathrm{H}_{2} \mathrm{O}_{2}+\mathrm{O}_{2}\right)$, a strong oxidant which readily diffuses across membranes [8-10]. By the Fenton reaction, hydrogen peroxide eventually yields the reactive hydroxyl radical $\left(\mathrm{OH}^{\circ}\right)$, which in turn disrupts cellular membranes [11]. It has been frequently stated that $\mathrm{OH}$ and singlet oxygen are the damaging agents $[12,13]$ that promote lipid peroxidation as a putative mechanism for paraquat toxicity [14].

It is often accepted that NADPH-cytochrome c reductase of microsomes generates free radicals under 
the action of paraquat $[13,15]$. The cytochrome P-450 and radical oxygen species are probably involved in efficient NADPH-dependent lipid peroxidation in rat adrenal cortex mitochondria [16]. NADPH-dependent lipid peroxidation occurs by the action of $\mathrm{O}_{2}{ }^{-}$and iron, and it is inhibited by $\mathrm{H}_{2} \mathrm{O}_{2}$ in human placental mitochondria [17], with a stimulatory degradative effect of paraquat. Conversely, other authors [18] consider that paraquat action, on lung lipids, is not consistent with peroxidation.

Lipid peroxidation in bovine adrenal cortex mitochondria is activated by $\mathrm{Fe}^{2+}$ and enzymatically stimulated by NADPH, an action strongly inhibited by paraquat [16]. Rat liver mitochondria are strongly affected by paraquat, stimulating state 4 respiration, without significant effect on state 3 respiration, and inducing lipid peroxidation $[19,20]$. It is also considered that paraquat accepts electrons via complex I and gets converted into the paraquat radical [21]. A novel redox system participating in paraquat toxicity is also related with free radicals produced by outer membrane mitochondrial NADH-quinone oxidoreductase [22]. On the other hand, Nagata et al. [23] have shown that mitochondria from bovine heart and yeast can catalyze the reduction of $\mathrm{NAD}^{+}$by reduced paraquat, the cofactor affording a protective action against paraquat toxicity.

In contrast to studies with animal fractions, the published studies in plants never used mitochondrial fractions to directly evaluate the effect of paraquat. Although Bowler et al. [24], using plant leaves, showed that mitochondrial metabolism participates in paraquat's damaging effects. They observed that, while paraquat affects cells under dark conditions, leaves having mitochondrial MnSOD-overproduction showed cellular protection against oxidative damage induced by paraquat. This finding, although obtained only while using tissues, suggests the involvement of mitochondria on the mechanism of paraquat toxicity.

The goal of this work was to find out the effects of paraquat and its radical on potato tuber mitochondria and rat liver mitochondria, which exhibit different sensitivities to protective antioxidant agents and different susceptibilities to oxidative stress. Protection against paraquat toxicity may involve neutralization of the reactive paraquat radical by the NADH redox system (matrix side) of the potato tuber mitochondrial complex I. Rat liver mitochondria may not have this protection. These results were obtained by following relevant bioenergetic parameters of mitochondria, e.g. the protonmotive force, respiration activities (state 4, state 3 , and uncoupled respiration). Furthermore, SOD activities and lipid peroxidation assays were also implemented.

\section{MATERIALS AND METHODS}

\section{Preparation of Mitochondria}

Fresh potato tubers (Solanum tuberosum L.) were obtained from the local market. Mitochondria were isolated and purified according to a procedure involving Percoll gradient centrifugation as a terminal purification step [25], except that 22\% Percoll (instead of 28\%) was used. The mitochondrial fraction was collected from the Percoll gradient with a Pasteur pipette and washed twice, by centrifugation, at $30000 \times g$ for $5 \mathrm{~min}$ in a washing medium (medium A) containing $250 \mathrm{mM}$ mannitol, $0.1 \%$ BSA and $10 \mathrm{mM}$ Hepes (pH 7.2). The pellet was gently resuspended in medium A, at a protein concentration of $20-30 \mathrm{mg} / \mathrm{mL}$. Rat liver mitochondria were obtained by differential centrifugations [19]. Protein was determined by the procedure of Bradford [26], calibrated with BSA standards.

\section{Measurement of Respiratory Activities}

Oxygen consumption was monitored with a Clark oxygen electrode, at $25^{\circ} \mathrm{C}$. Polarographic measurements were performed in a $1.5 \mathrm{~mL}$ reaction medium (medium B) containing $0.25 \mathrm{M}$ sucrose, $30 \mathrm{mM} \mathrm{KCl}$, $15 \mathrm{mM}$ Hepes (pH 7.2), $20 \mathrm{mM} \mathrm{KCl}, 5 \mathrm{mM} \mathrm{K}_{2} \mathrm{HPO}_{4}$, $2 \mathrm{mM} \mathrm{MgCl}_{2}, 0.2 \mathrm{mM}$ EDTA, and $0.05 \%$ BSA. State 3 was elicited by adding $1.0 \mathrm{mM}$ ADP and uncoupled respiration by adding $1 \mu \mathrm{M}$ carbonyl cyanide $p$-trifluoromethoxyphenyl-hydrazone (FCCP). Respiration rates were calculated by assuming an oxygen concentration of $250 \mathrm{nmol} / \mathrm{mL}$ in the experimental medium at $25^{\circ} \mathrm{C}$.

The mitochondrial transmembrane electric potential $(\Delta \psi)$ was monitored with a tetraphenyl phosphonium $\left(\mathrm{TPP}^{+}\right)$-electrode prepared according to Kamo et al. [27]. All experiments were carried out in an open vessel at $25^{\circ} \mathrm{C}$, with efficient magnetic stirring in $1 \mathrm{~mL}$ of medium B, supplemented with $3 \mu \mathrm{M} \mathrm{TPP}{ }^{+}$. About $0.3 \mathrm{mg} / \mathrm{mL}$ of mitochondrial protein were used in the reaction medium supplemented with the additions indicated in the legends of figures.

The relative $\Delta \psi$ inhibition by paraquat (slow timedependent effect), along $10 \mathrm{~min}$ respiration $(A)$, was determined by the formula $A=100-(C / D) \times 100$, and the total $\Delta \psi$ inhibition by paraquat (sharp initial effect plus slow time-dependent effect), after $10 \mathrm{~min}$ respiration $(B)$, was determined by the formula, $B=100-$ $\left(C / D^{*}\right) \times 100$, where $C$ is $\Delta \psi$ after 10 min respiration for each paraquat concentration, $D$ is the maximum $\Delta \psi$ reached in the assay, and $D^{*}$ is the $\Delta \psi$ after 10 min respiration in the absence of paraquat.

Membrane potential was calculated using the equation of Kamo et al. [27], where the deflection of the 
$\mathrm{TPP}^{+}$-electrode potential $(\Delta E)$ was determined according to the size of the trace collapsed as a consequence of valinomycin-induced $\mathrm{K}^{+}$diffusion, by using $75 \mathrm{ng} / \mathrm{mL}$ valinomycin at the end of each assay.

\section{Superoxide Dismutase Activity}

Superoxide dismutase (SOD) activity was determined according to the method of Payá et al. [28], using $0.1 \mathrm{mM}$ hypoxanthine and $0.025 \mathrm{U} / \mathrm{mL}$ xanthine oxidase as $\mathrm{O}_{2}{ }^{--}$source and $0.1 \mathrm{mM}$ nitroblue tetrazolium (NBT) as $\mathrm{O}_{2}{ }^{-}$scavenger in $\mathrm{KH}_{2} \mathrm{PO}_{4}$ buffer $(\mathrm{pH}$ 8.1). NBT reduction was monitored at $560 \mathrm{~nm}$ in a PerkinElmer Lambda 6 spectrophotometer. SOD activity was determined for mitochondrial fractions in the presence and absence of $0.025 \%(\mathrm{v} / \mathrm{v})$ Triton $\mathrm{X}-100$, in $3 \mathrm{~mL}$ of assay mixture.

\section{Determination of Thiobarbituric Acid Reactive Products}

Lipid peroxidation was evaluated as thiobarbituric acid reactive products, as described previously [29]. The amount of reactive products formed was calculated by using an extinction coefficient of $165 \mathrm{mM}^{-1} \mathrm{~cm}^{-1}$ at $530 \mathrm{~nm}(30)$.

\section{$\alpha$-Tocopherol Assay}

The assay was performed with $3 \mathrm{mg}$ of each mitochondrial fraction $(20-30 \mathrm{mg} / \mathrm{mL})$ in a solution containing $1.5 \mathrm{~mL}$ SDS (sodium dodccyl sulphate) $(10 \mathrm{mM}), 2 \mathrm{~mL}$ ethanol, $2 \mathrm{~mL} n$-hexane, and $50 \mu \mathrm{L} \mathrm{KCl}(3 \mathrm{M})$. The mixture was vigorously vortexed for $3 \mathrm{~min}$ and filtered (Whatman No. 1). The mixture was centrifuged at room temperature for $5 \mathrm{~min}$ at $1500 \times g$. The upper phase, partitioned in $n$-hexane, was dried under vacuum at $50^{\circ} \mathrm{C}$. The residue was hermetically kept at $-80^{\circ} \mathrm{C}$ until subjected to chromatography by redissolving in $n$-hexane for reverse phase HPLC analysis [31], on Spherisorb S10w, $4.6 \times 200 \mathrm{~mm}$, equilibrated with a mixture of $n$-hexane with $0.9 \%$ methanol at a flow of $1.5 \mathrm{~mL} / \mathrm{min}$. UV absorption of the column eluate was monitored at $287 \mathrm{~nm}$ and recorded as peaks. Calculations were performed from peak areas calibrated with standards of pure $\alpha$-tocopherol [32,33].

\section{Treatment of Data}

Results are presented as fractions of controls for at least three independent experiments. Direct records representative of at least three independent experiments are also displayed.

\section{Chemicals}

All reagents were of analytical grade. The herbicide paraquat was purchased from Fluka ChemicaBiochemica and used as aqueous solution. All the other compounds were from Sigma.

\section{RESULTS}

\section{The Use of TPP $^{+}$-Electrode for Fast Detection of Paraquat Toxicity}

The transmembrane mitochondrial electric potential $(\Delta \psi)$ was estimated with several methodologies $[27,34]$ that are useful to evaluate bioenergetic toxicity exerted by xenobiotics. The $\mathrm{TPP}^{+}$-electrode is highly sensitive to small effects but is prone to artifacts (e.g. binding of compounds to the $\mathrm{TPP}^{+}$membrane), and experimental conditions have to be carefully controlled. Hence, the signal was always calibrated by adding valinomycin $(1 \mu \mathrm{M})$ at the end of each assay to establish the reference baseline (full collapse of the transmembrane potential).

The $\mathrm{TPP}^{+}$-electrode used for monitoring $\Delta \psi$ of bioenergized mitochondria in the presence of paraquat induced, by itself, an artifactual $\Delta \psi$ dissipation (Figure 1A), evident after several hours of continuous

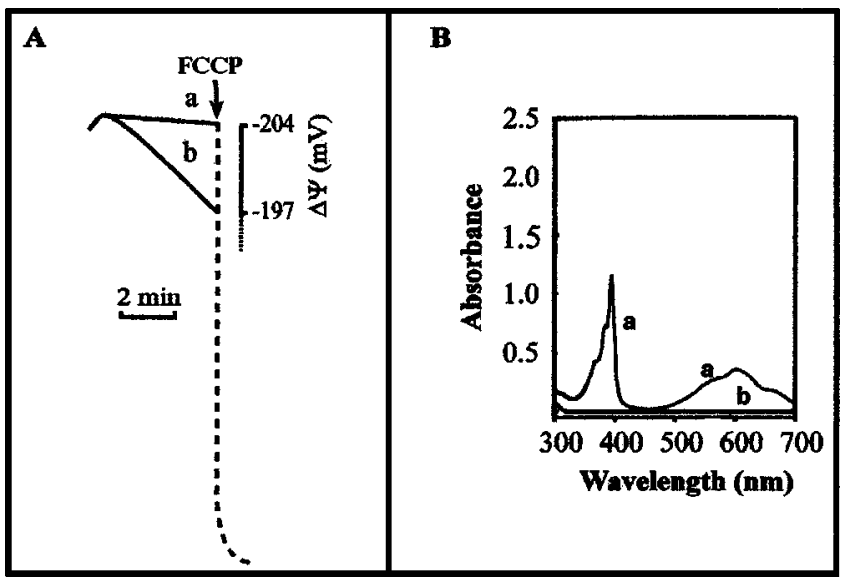

FIGURE 1. Artifactual membrane potential $(\Delta \psi)$ dissipation induced by paraquat on potato mitochondria. (A) $\mathrm{TPP}^{+}$-electrode traces of succinate-supported mitochondrial $\Delta \psi$ using noncontaminated $\mathrm{TPP}^{+}$-electrode (a), and an electrode which was contaminated by the paraquat used in the assays (b). FCCP indicates addition of $1 \mu \mathrm{M}$ FCCP. (B) Spectra obtained from the bluish electrolyte inside the contaminated $\mathrm{TPP}^{+}$-electrode (a), and from the paraquat solution plus $\mathrm{TPP}^{+}(\mathrm{b})$ 
use. A bluish color was increasingly apparent inside the contaminated electrode, where only a colorless $\mathrm{TPP}^{+}$ solution was to be present. The color resulted from the presence of the paraquat radical, which was confirmed by spectrophotometry of the contents of the contaminated $\mathrm{TPP}^{+}$-electrode. A characteristic peak at $605 \mathrm{~nm}$ typical of paraquat radical [8] was detected. In these conditions, the contaminated $\mathrm{TPP}^{+}-$ electrode induced a continuous artifactual dissipation of $\Delta \psi$ (Figure 1A, trace b) in succinate or ascorbate/ $N, N, N^{\prime}, N^{\prime}$-tetramethyl- $p$-phenylenediamine (TMPD) energized mitochondria.

In spite of limitations, the controlled use of the $\mathrm{TPP}^{+}$-electrode is a very sensitive and reliable method to detect and evaluate small $\Delta \psi$ changes. Furthermore, the electrode contamination by paraquat radical was a direct visual evidence of paraquat radical formation occurring as a consequence of mitochondrial respiration, in agreement with previously reported data $[35,36]$.

\section{Effects of Paraquat on Succinate-Dependent Respiration}

The $\Delta \psi$ coupled to succinate-supported respiration was significantly more affected by paraquat (Figure 2) in rat liver mitochondria than in potato tuber mitochondria. The $\Delta \psi$ elicited by rat liver mitochondria respiring succinate was dissipated along the respiration period by exposure to concentrations higher than $1 \mathrm{mM}$ paraquat (Figure 2). This effect increased as a function

$\mathbf{A}$

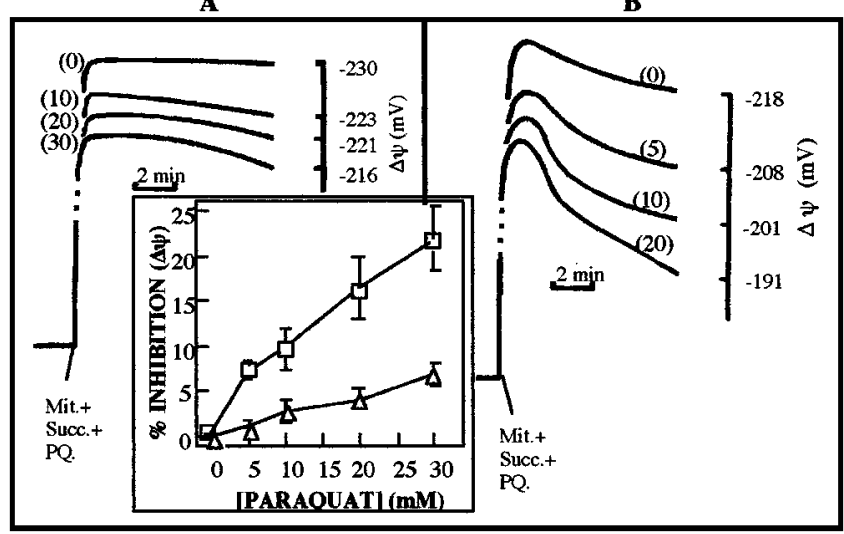

FIGURE 2. Effect of paraquat on potato tuber (A) and rat liver (B) mitochondrial $\Delta \psi$ during respiration of succinate. Concentrations of paraquat (in $\mathrm{mM}$ ) are given in parenthesis. Inset, Total effect of paraquat on the $\Delta \psi$ promoted by succinate-supported respiration of potato tuber mitochondria $(\Delta)$ and rat liver mitochondria, $(\square)$ as a function of paraquat concentration. The depicted values were obtained as percentage of $\Delta \psi$ inhibition by paraquat after $10 \mathrm{~min}$ respiration. The assays were performed as described in Materials and Methods. of the herbicide concentration, and it was possible to distinguish this as occurring in two phases. Paraquat induced an immediate small decrease in $\Delta \psi$, probably not physiologically important, since it was detected only with higher concentrations of paraquat used for shortterm effects in vitro. It can also be related with an artifact of the $\mathrm{TPP}^{+}$-electrode, as consequence of binding to its membrane. Therefore, we did not consider this effect in the mechanism of paraquat toxicity on mitochondria. After the initial fast effect, a slow dissipation of $\Delta \psi$ proceeded with time (Figure 2). Increasing concentrations of paraquat promoted higher rates of $\Delta \psi$ dissipation with time. Potato tuber mitochondria (Figure 2A), as compared with rat mitochondria (Figure 2B), were similarly affected, but they were significantly less sensitive. Clearly, higher levels of $\Delta \psi$ dissipation were observed with rat than with potato mitochondria (Figure 2, inset). Additionally, for each mitochondrial type, the sensitivity of paraquat differed from preparation to preparation. The variability range was wider for rat mitochondria (Figure 2, inset, error bars), suggesting that the paraquat toxicity mechanism was dependent on variable factors of the mitochondrial fractions.

Simultaneous with $\Delta \psi$ dissipation, we detected that a progressive stimulation of state $4 \mathrm{O}_{2}$ consumption (Figure 3) occured as a function of paraquat concentration, indicating that permeabilization of the mitochondrial membrane resulted in proton leak, accelerating oxygen consumption.

Respiration associated with ADP phosphorylation (state 3) was not significantly affected, with only a small

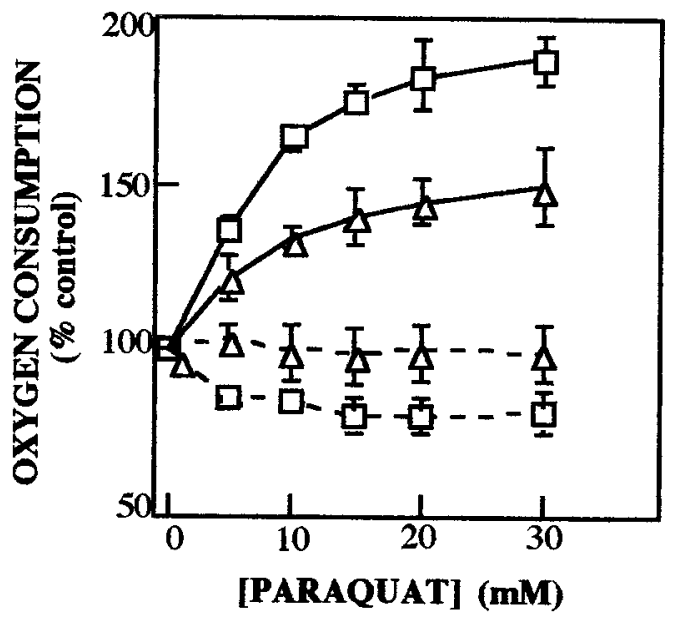

FIGURE 3. Effect of paraquat on state 4 and state 3 respiration in potato tuber mitochondria $(\Delta)$ and rat liver mitochondria $(\square)$. Control values for succinate $(10 \mathrm{mM})$ supported respiration are given in nmol $\mathrm{O}_{2}$ (mg protein $)^{-1} \mathrm{~min}^{-1}$. State 4 (solid lines): potato, $48.4 \pm 16.1$; rat, $23.3 \pm 10.2$. State 3 (dashed lines): potato, $183.5 \pm 70.3$; rat, $84.9 \pm$ 24.6. State 3 was elicited by adding $1 \mathrm{mM}$ ADP. 
inhibitory action (ca. 20\%) being noticed for rat liver mitochondria (Figure 3). Oxidative phosphorylation of potato tuber mitochondria, as indirectly inferred from depolarization-repolarization kinetics after ADP addition, was not significantly affected by paraquat (up to $40 \mathrm{mM}$ ) in short-term experiments (results not shown).

Data taken together were indicative of a protonophoric effect by paraquat on the mitochondrial membranes. This could not be demonstrated by swelling experiments in K-acetate, which are dependent on protonophoric action (results not shown). Unexpectedly, paraquat inhibited the swelling effect dependent on protonophoric action.

\section{Influence of the Respiratory Substrates on the Efficiency of Paraquat}

Figure 4 shows that paraquat dissipates plant mitochondrial $\Delta \psi$ promoted by the tested respiratory substrates, succinate, exogenous $\mathrm{NADH}$, and ascorbate/TMPD, except those for complex I (Figure 4A). $\mathrm{TPP}^{+}$-electrode traces clearly show that the $\Delta \psi$ is not affected during the assay time, even in the presence of $40 \mathrm{mM}$ paraquat, when potato mitochondria are respiring complex I substrates but not succinate (Figure 4B). According to other published results, with bovine heart mitochondria and yeast mitochondria [23,37], $\mathrm{NAD}^{+}$is converted into NADH by action of $\mathrm{PQ}^{+}$, which, in turn, transforms into the harmless paraquat divalent cation [23].

Addition of malate/pyruvate and $\mathrm{NAD}^{+}$to plant mitochondria respiring succinate did not afford

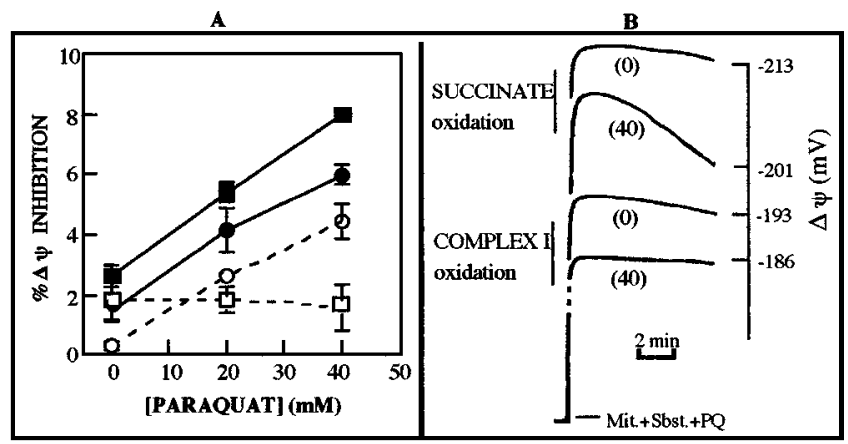

FIGURE 4. Differences on the slow time-dependent effects of paraquat on the $\Delta \psi$ promoted by potato tuber mitochondria oxidizing different substrates. (A) Inhibition of different paraquat concentrations on the $\Delta \psi$ promoted by $0.75 / 10 \mathrm{mM}$ TMPD/ascorbate $(\boldsymbol{\square}) ; 1 \mathrm{mM}$ exogenous NADH $(\bullet) ; 10 \mathrm{mM}$ succinate $(\mathrm{O})$; and 30/6/0.5/0.5 mM malate/pyruvate/ $\mathrm{NAD}^{+} /$thiamine pyrophosphate (ם). (B) Evolution of $\Delta \psi$ promoted by succinate and complex I respiration with or without paraquat $(40 \mathrm{mM})$. Experiments were performed as indicated in Figure 2. In A, values are the means \pm S.E. of at least 3 independent experiments. In $B$, the depicted traces are representative of four independent assays.

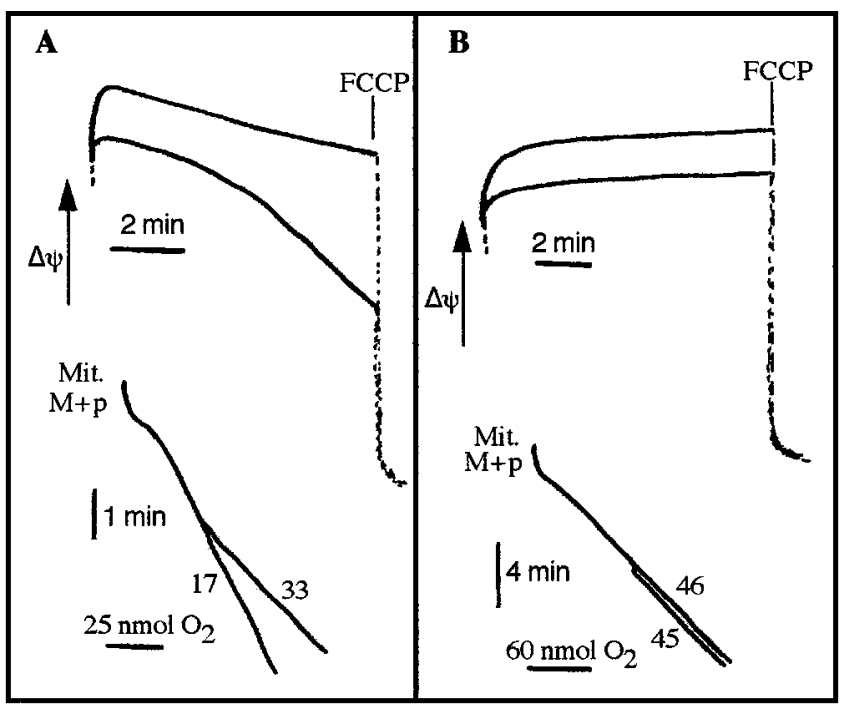

FIGURE 5. Simultaneous effects of paraquat on both $\Delta \psi$ and corresponding $\mathrm{O}_{2}$ consumption of complex-I-dependent respiration, of rat liver mitochondria (A) and potato tuber mitochondria (B). $\mathrm{TPP}^{+}$-electrode traces (upper) were recorded in the presence of the paraquat concentration expressed in parenthesis, in $\mathrm{mM} . \mathrm{O}_{2}$ consumption traces (lower), in the absence (a) and in the presence (b) of paraquat, indicate numbers expressing activities in $\mathrm{mol}_{2}(\mathrm{mg}$ protein $)^{-1} \mathrm{~min}^{-1}$. FCCP, indicates addition of $1 \mu \mathrm{M}$ FCCP.

any protection against paraquat toxicity (results not shown), although $\mathrm{NAD}^{+}$and complex I substrates are both transported into the plant mitochondria. This suggests that the referred protective effect is assigned to the exclusive action of enzymatic activity related to complex I rather than to a reaction of the substrates, by themselves, with $\mathrm{PQ}^{+}$. Figure 5 shows that $20 \mathrm{mM}$ paraquat considerably dissipates complex-I-dependent $\Delta \psi$ of liver mitochondria (Figure 5A) during the assay time, but not potato tuber mitochondria, even at $40 \mathrm{mM}$ paraquat (Figure 6B). In agreement with these results,

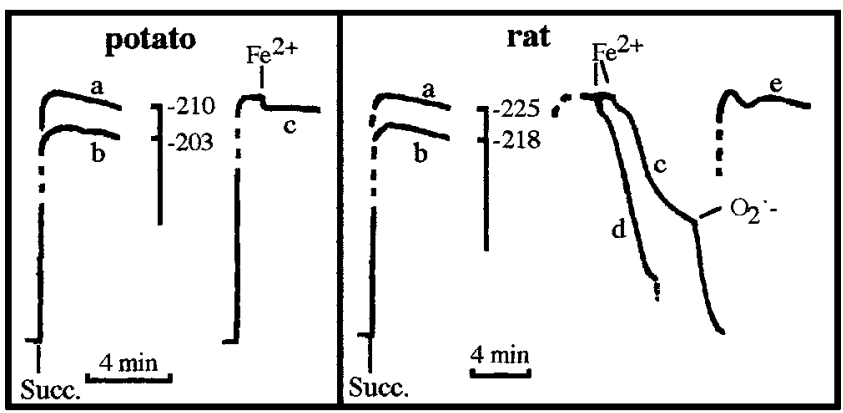

FIGURE 6. Comparative sensitivities of $\Delta \psi$ promoted by potato and rat mitochondria to paraquat and artificial oxidative stress induced by adding $\mathrm{H}_{2} \mathrm{O}_{2}, \mathrm{Fe}^{2+}$, and an $\mathrm{O}_{2}{ }^{--}$generating system. Succ., addition of $10 \mathrm{mM}$ succinate to start the assay. Control (a); addition of $30 \mathrm{mM}$ paraquat (b); $240 \mu \mathrm{M} \mathrm{Fe}^{2+}$ and further addition of $\mathrm{O}_{2}{ }^{--}$, where indicated (c); $300 \mu \mathrm{M} \mathrm{Fe}^{2+}$ (d); $2 \mathrm{mM} \mathrm{H}_{2} \mathrm{O}_{2}+\mathrm{O}_{2}{ }^{--}$(e). 
state $4 \mathrm{O}_{2}$ consumption was stimulated by paraquat in assays with rat mitochondria but remained unaffected with potato tuber mitochondria (Figure $6 \mathrm{~A}$ and $\mathrm{B}$, lower records). These results indicate that the paraquat toxicity, which depends on the formation of $\mathrm{PQ}^{+}$, acts on membrane permeability.

\section{Different Sensitivities of Mitochondria to Paraquat Oxidative Stress As a Function of the Level of Antioxidant Agents}

Because paraquat toxicity is believed to arise from oxidative stress, resistance of mitochondria to controlled oxidative stress was investigated. An artificial $\mathrm{O}_{2}{ }^{-}$generating system (hypoxanthine/xanthine oxidase), $\mathrm{H}_{2} \mathrm{O}_{2}$, and $\mathrm{Fe}^{2+}$ were added together or separately to mitochondria respiring succinate. Addition of $\mathrm{O}_{2}{ }^{-}$and $\mathrm{H}_{2} \mathrm{O}_{2}$ did not affect $\Delta \psi$, but, in rat liver mitochondria, a rapid $\Delta \psi$ dissipation was observed upon addition of relatively high concentrations of $\mathrm{Fe}^{2+}(240$ or $300 \mu \mathrm{M}$ ) even without $\mathrm{O}_{2}{ }^{-}$and $\mathrm{H}_{2} \mathrm{O}_{2}$ (Figure 6). After $\mathrm{Fe}^{2+}$ addition, rat liver mitochondria became very sensitive to the presence of $\mathrm{O}_{2}{ }^{--}$. However, potato mitochondria were not affected by additions of $\mathrm{O}_{2}{ }^{--}$and $\mathrm{H}_{2} \mathrm{O}_{2}$ (2 mM), either separately or together (data not shown), and even if added with $240 \mu \mathrm{M} \mathrm{Fe}^{2+}$ (Figure 6).

Attempting to justify the different sensitivities of plant and animal mitochondria, we evaluated the scavenging capacity for $\mathrm{O}_{2}{ }^{--}$of both types of mitochondria, considering that superoxide production can be an intermediate for paraquat toxicity. Potato mitochondria exhibit a superoxide dismutase activity significantly stronger than rat liver mitochondria (Figure 7). This

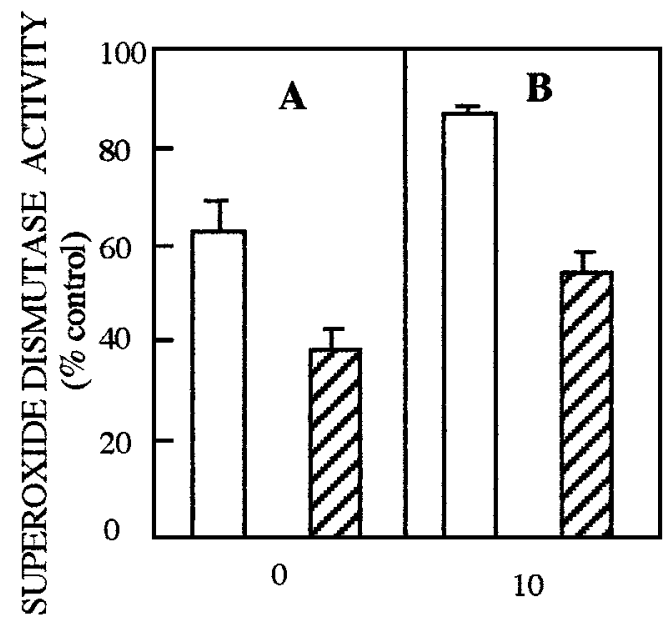

[PARAQUAT] (mM)

FIGURE 7. Scavenging of superoxide anions by potato tuber mitochondria $(\square)$, and rat liver mitochondria $(\mathbb{Z})$, in the absence (A) and in the presence of $10 \mathrm{mM}$ paraquat (B). The assays were performed as described in Materials and Methods.

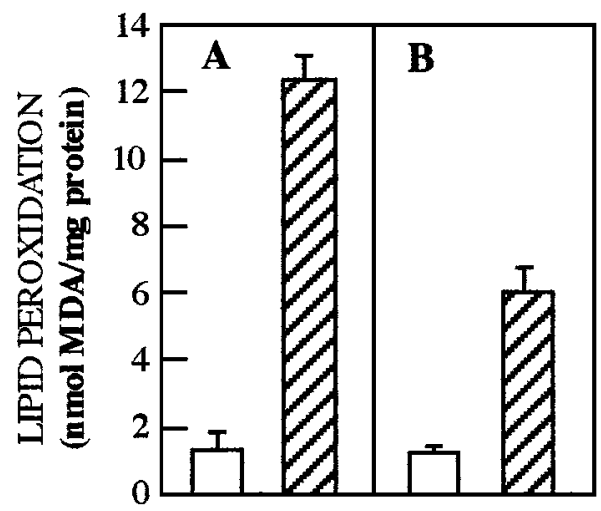

FIGURE 8. Effect of paraquat on the lipid peroxidation, as detected by malondialdehyde (MDA) production, of potato tuber mitochondria $(\square)$ and rat liver mitochondria ( $\square \boldsymbol{Z})$. The mitochondrial protein $(1 \mathrm{mg})$, plus succinate, was incubated in the presence of $30 \mathrm{mM}$ paraquat, at $30^{\circ} \mathrm{C}$ for $10 \mathrm{~min}$, in the absence (A) and in the presence (B) of antimycin $(1 \mu \mathrm{M})$.

difference may in part explain the protection of plant membranes against lipid peroxidation promoted by paraquat, considering that $\mathrm{O}_{2} \cdot{ }^{-}$is involved in the peroxidation process.

Lipid peroxidation was always higher in rat liver mitochondria than potato tuber mitochondria, either in conditions of active or inhibited respiration (Figure 8). Inhibition of succinate-supported respiration by antimycin A reduced malondialdehyde formation to less than $50 \%$ in rat liver mitochondria, not affecting potato tuber mitochondria, which was very resistant to lipid peroxidation induced by paraquat.

In general, data on lipid peroxidation suggest the presence of protective agents (antioxidants) in higher concentrations in potato as compared to those in liver mitochondria. Indeed, the relative amount of $\alpha$-tocopherol in potato mitochondria was $2.92 \pm$ $0.78 \mathrm{nmol}$ (mg protein $)^{-1}$, and the value for rat was $55 \%$ of this. Other antioxidants existing in potato cells, namely carotenoids and phenolic compounds, are supposed to be excluded from the purified mitochondrial fractions, and therefore do not explain the increased resistance to peroxidation of plant mitochondria.

\section{DISCUSSION}

Mitochondria are sites for production of oxygen free radicals, which are known to be involved in a variety of damaging effects [38-43]. In spite of a large number of reports on the mechanisms of paraquat toxicity, discrepancies of interpretation are frequent, requiring additional research to clarify the mechanisms of action. It has been reported that the target for paraquat 
toxicity in animal materials is the NADPH-dependent lipid peroxidation of microsomal lipids [13,15], but the mitochondrial respiratory chain is also a possibility $[2,16,17,20,23]$, including the outer membrane mitochondrial NADH-quinone oxidoreductase [22].

Most of the studies with plants describe the effects of paraquat on chloroplasts [1,3-6,9-11], with photosystem I as the target for herbicide toxicity. However, plant mitochondria, like animal mitochondria $[2,16,17,20,22,23,35,36]$, can also be a target.

It has been shown that paraquat enters plant cells [44] and continuously accepts electrons from one of the electron carriers [23,35], diverting part of the electron flow to the putative production of $\mathrm{O}_{2}{ }^{--}$and $\mathrm{H}_{2} \mathrm{O}_{2}$ [12].

Interestingly, the artifactual results observed with our contaminated electrodes (Figure 1) demonstrate effective production of the $\mathrm{PQ}^{+}$, this probably being the first step of the toxicological mechanism responsible for the time-dependent $\Delta \psi$ dissipation in mitochondria. The crossing of the $\mathrm{TPP}^{+}$-electrode membrane by $\mathrm{PQ}^{+}$(Figure 1) indicates that the radical can cross the mitochondrial membrane.

Whether on the outside or inside of the matrix, $\mathrm{PQ}^{+}$ reacts with $\mathrm{O}_{2}$, yielding $\mathrm{O}_{2}{ }^{--}$[7]. In turn, the $\mathrm{O}_{2}{ }^{--}$generated in the matrix can be dismutated by Mn-SOD into $\mathrm{H}_{2} \mathrm{O}_{2}$ [8-10]. This compound, through the Fenton reaction, promptly generates $\mathrm{OH}$, a very reactive and damaging radical [11-14]. Redox cycling of paraquat may also occur outside, with formation of $\mathrm{O}_{2}{ }^{-}$and dismutation into $\mathrm{H}_{2} \mathrm{O}_{2}$ by a mitochondrial $\mathrm{Cu}, \mathrm{Zn}-$ superoxide dismutase existing in the intermembrane space [45]. We assayed artificial production of high superoxide levels (Figure 7) in the presence of respiring mitochondria and demonstrated that no $\Delta \psi$ dissipation occurs. Therefore, if paraquat radical induces $\mathrm{O}_{2}{ }^{--}$ production as an essential step for paraquat toxicity, it may be assumed that redox cycling of paraquat outside the mitochondria is relatively harmless, as compared with the redox cycling in the matrix, probably as consequence of a fast transference of electrons from $\mathrm{O}_{2} \cdot{ }^{-}$to cytochrome $\mathrm{c}$ (and further to $\mathrm{O}_{2}$ in cytochrome oxidase), with regeneration of $\mathrm{O}_{2}$ [46].

Paraquat induced a time-dependent dissipation of the $\Delta \psi$ produced by respiration with succinate, exogenous NADH, and TMPD/ascorbate, but this effect was not detected in potato tuber mitochondria respiring complex I substrates. Knowing that TMPD/ascorbate promotes the activity of cytochrome c oxidase alone, and considering that this respiratory complex is common to the other refered substrates, at least part of the effects is related to the cytochrome c oxidase activity. On the other hand, the complex I may afford protection for the paraquat effects on plant mitochondria. These findings are at variance with authors who consider complex I responsible for paraquat effects on animal mitochondria $[35,36]$. However, Nagata et al. [23] demonstrated (in agreement with our results in plant mitochondria) that bovine heart mitochondria and yeast mitochondria catalyse the reduction of $\mathrm{NAD}^{+}$ to NADH at the expense of the paraquat radical. They could not establish whether enzymes of the respiratory chain or other enzymes of the mitochondria are involved. We show that mitochondrial complex I respiration of potato tuber, in contrast to that in rat liver, avoids from deleterious effects dependent on the paraquat presence. Our data indicate that the herbicide looses the capacity of $\Delta \psi$ dissipation in potato respiration supported by complex I. It was also noticed that the presence of complex I substrates or the addition of $\mathrm{NAD}^{+}$ (that in plants can enter the mitochondrial membranes), simultaneously with succinate, no longer afforded protection against paraquat. Therefore, the protective action of plant complex I respiration is not assigned to the substrates or cofactors, by themselves, but instead it is related to the redox activity of complex I. It should be remembered that, in contrast with rat mitochondria, exogenous NADH can be oxidized by the so-called plant external NADH-dehydrogenase, producing $\mathrm{NAD}^{+}$, without protective action against the paraquat radical (Figures 4 and 5). This indicates that the protective action, involving $\mathrm{NAD}^{+}$, is exclusive to the complex I activity. Considering that this complex I respirationdependent protection involves $\mathrm{PQ}^{+}$reduction, as confirmed by Nagata et al. [23], penetration of $\mathrm{PQ}^{+}$to the inside of mitochondria is essential to explain the results.

Attempting to understand the different sensitivities of plant and animal mitochondria to paraquat, we looked for direct effects of oxidative stress agents on mitochondria. Exogenously added $\mathrm{O}_{2}{ }^{-\cdot}$ and $\mathrm{H}_{2} \mathrm{O}_{2}$ produced no significant effects on mitochondrial succinatesupported $\Delta \psi$. However, high $\mathrm{Fe}^{2+}(300 \mu \mathrm{M})$ depressed succinate-dependent $\Delta \psi$ of rat liver mitochondria, in contrast with the insensitivity of plant mitochondria (Figure 6). The differences may relate to the relative concentrations of protective agents in plant mitochondria, namely, the very effective lipid antioxidant $\alpha$ tocopherol [32] present at higher concentrations in plant material, particularly potato tuber mitochondria [31]. This explains the higher resistance to paraquat induced lipid peroxidation in potato tuber mitochondria, as compared to that the rat liver mitochondria. These findings and interpretations fully agree with the enhanced paraquat toxicity described for mice deficient in $\alpha$ tocopherol [47]. Lipid peroxidation data of both kinds of mitochondria (Figure 8) are correlated with the levels of paraquat toxicity.

Bowler et al. [24] demonstrated increased resistance to paraquat in transgenic tobacco plants overexpressing mitochondrial Mn-SOD in leaf cells, but a direct 
proof for the involvement of mitochondria was never presented. As regards this, our data are consistent with the higher resistance of plant mitochondria to paraquat $\Delta \psi$ dissipation (Figure 2 ) as a consequence of its higher level of $\mathrm{O}_{2}{ }^{--}$scavenging action (Figure 7), by reference to the level in animal mitochondria. Therefore, a higher activity of mitochondrial SOD may provide increased resistance against oxidative stress promoted by paraquat. High levels of ascorbyl radical reductase in potato tuber mitochondria [48] also protects against the product of SOD activity, namely $\mathrm{H}_{2} \mathrm{O}_{2}$.

In conclusion, this work adds new information on the mechanism of paraquat action, identifying different sensitivities of plant and animal mitochondria, in correlation with different properties of mitochondrial antioxidant protective agents. Potato tuber mitochondria, as to compared with rat liver mitochondria, are significantly more protected against paraquat stress as consequence of higher levels of antioxidant agents, namely $\alpha$-tocopherol and SOD. Furthermore, it has been shown that plant mitochondrial complex I activity, as in bovine heart mitochondria and yeast mitochondria [23], but not liver mitochondria, neutralizes paraquat radical toxicity, in contrast to the other respiratory complexes. Finally, it appears that the mitochondrial mechanism of paraquat toxicity involves the entrance of paraquat radical into the mitochondrial matrix.

\section{REFERENCES}

1. Cremlyn JR. Agrochemicals: Preparation and Mode of Action. Chichester, England: Wiley, 1991.

2. Sata T, Takeshige K, Takayanagi R, Minakami S. Lipid peroxidation by bovine heart submitochondrial particles stimulated by 1,1'-dimethyl-bipyridy (paraquat). Biochem Pharmacol 1983;32:13-19.

3. Stevens JT, Sumner DD. Herbicides. In: Hayes WJ, Laws ER, editors. Handbook of Pesticide Toxicology; 1991. pp 1317-1408.

4. Corbett JR, Wright K, Baillie AC. The Biochemical Mode of Action of Pesticides, 2nd edition. London: Academic Press; 1984. pp 87-89.

5. Fujii T, Yokoyama E, Inoue K, Sakurai H. The sites of electron donation of photosystem I to methyl viologen. Biochem Biophys Acta 1990;1015:41-48.

6. Preston C. Resistance to photosystem I disruption herbicides. In: Powles SB, Holtum JAM, editors. Herbicide Resistance in Plants: Biology and Biochemistry. Boca Raton, FL: CRC Press; 1994. pp 61-82.

7. Hassan HM, Fridovich I. Paraquat and Escherichia coli. Mechanism of production of extracellular superoxide radical. J Biol Chem 1979;254:10846-10852.

8. Farrington JA, Ebert M, Land EJ, Fletcher K. Bipyridylium quaternary salts and related compounds. V. Pulse radiolysis studies of the reaction of paraquat radical with oxygen. Implications for the mode of action of bipyridyl herbicides. Biochim Biophys Acta 1973;314:372-381.
9. Miller RW, MacDowall FDH. The tiron free radical as a sensitive indicator of chloroplastic photoautoxidation. Biochim Biophys Acta 1975;387:176-187.

10. Harbour JR, Bolton JR. Superoxide formation in spinach chloroplasts: Electron spin resonance detection by spin trapping. Biochem Biophys Res Commun 1975;64:803807.

11. Babbs CF, Pham JA, Coolbaugh RC. Lethal hydroxyl radical production in paraquat-treated plants. Plant Physiol 1989;90:1267-1270.

12. McCord JM, Day ED, Jr. Superoxide-dependent production of hydroxyl radical catalyzed by iron-EDTA complex. FEBS Lett 1978;86:139-142.

13. Bus JS, Aust SD, Gibson JE. Superoxide- and singlet oxygen-catalyzed lipid peroxidation as a possible mechanism for paraquat (methyl viologen) toxicity. Biochem Biophys Res Commun 1974;58:749-755.

14. Castro GD, Lopez A, Castro JA. Evidence for hydroxyl free radical formation during paraquat exposure but not for nifurtimox liver microsomal biotransformation. A dimethylsulfoxide scavenging study. Arch Toxicol 1988;62:355-358.

15. Bus JS, Cagen SZ, Olgaard M, Gibson JE. A mechanism of paraquat toxicity in mice and rats. Toxicol App Pharmacol 1976;35:501-513.

16. Klimek J, Schaap AP, Kimura T. Effect of paraquat on cytochrome P-450-dependent lipid peroxidation in bovine adrenal cortex mitochondria. Biochim Biophys Acta 1983;752:127-136.

17. Klimek J. The involvement of superoxide and iron ions in the NADPH-dependent lipid peroxidation in human placental mitochondria. Biochim Biophys Acta 1988;958:3139 .

18. Kornbrust DJ, Mavis RD. The effect of paraquat on microsomal lipid peroxidation in vitro and in vivo. Toxicol App Pharmacol 1980;53:323-332.

19. Palmeira CM, Moreno AJ, Madeira VMC. Mitochondrial bioenergetics is affected by the herbicide paraquat. Biochim Biophys Acta 1995;1229:187-192.

20. Thakar JH, Hassan MN. Effects of 1-methyl-1,2,4,6tetrahydropyridine (MPTP), cyperquat $\left(\mathrm{MPP}^{+}\right)$and paraquat on isolated mitochondria from rat striatum, cortex and liver. Life Sci 1988;43:143-149.

21. Fukushima T, Yamada K, Isobe A, Shiwaku K, Yamane Y. Mechanism of cytotoxicity of paraquat. I. NADH oxidation and paraquat radical formation via complex I. Exp Toxicol Pathol 1993;45:345-349.

22. Shimada H, Hirai K-I, Simamura E, Pan J. Mitochondrial $\mathrm{NADH}$-quinone oxidoreductase of the outer membrane is responsible for paraquat cytotoxicity in rat liver. Arch Biochem Biophys 1998;351:75-81.

23. Nagata S, Gunther H, Bader J, Simon H. Mitochondria catalyse the reduction of $\mathrm{NAD}^{+}$by reduced methyl viologen. FEBS Lett 1987;210:66-70.

24. Bowler M, Slooten L, Vandenbranden S, De Rycke R, Botterman J, Sybesma C, Van Montagu M, Inzé D. Manganese superoxide dismutase can reduce cellular damage mediated by oxygen radicals in transgenic plants. EMBO J 1991;10:1723-1732.

25. Neuburger M, Journet E-P, Bligny R, Carde J-P, Douce R. Purification of plant mitochondria by isopycnic centrifugation in density gradients of Percoll. Arch Biochem Biophys 1982;217:312-323.

26. Bradford MM. A rapid and sensitive method for the quantification of microgram quantities of protein utilizing 
the principle of protein-dye binding. Anal Biochem 1976;72:248-264.

27. Kamo N, Muratsugu M, Hongoh R, Kobatake $Y$. Membrane potential of mitochondria measured with an electrode sensitive to tetraphenyl phosphonium and relationship between proton electrochemical potential and phosphorylation potential in steady state. J Membr Biol 1979;49:105-121.

28. Payá M, Halliwell B, Hoult JRS. Interactions of a series of coumarins with reactive oxygen species. Biochem Pharmacol 1992;44:205-214.

29. Eriksson O, Pollesello P, Saris NEL. Inhibition of lipid peroxidation in isolated rat liver mitochondria by the general anesthetic propofol. Biochem Pharmacol 1992;44:391393.

30. Ottolenghi A. Interaction of ascorbic acid and mitochondrial lipids. Arch Biochem Biophys 1959;79:355-359.

31. Vatassery GT, Maynard UR, Hagen DF. Highperformance liquid chromatography of various tocopherols. J Chromat 1978;161:299-302.

32. Spychalla JP, Desborough SL. Superoxide dismutase, catalase, and $\alpha$-tocopherol content of stored potato tubers. Plant Physiol 1990;94:1214-1218.

33. Burton GW, Webb A, Ingold KV. A mild, rapid and efficient method of lipid extraction for use in determining vitamin E/lipid ratios. Lipids 1985;20:29-39.

34. Valle VGR, Pereira-da-Silva L, Vercesi AE. Undesirable feature of safranine as a probe for mitochondrial membrane potential. Biochem Biophys Res Commun 1986;135:189-195.

35. Fukushima T, Yamada K, Isobe A, Shiwaku K, Yamane Y. Mechanisms of cytotoxicity of paraquat: I. NADH oxidation and paraquat radical formation via complex. I. Exp Toxicol Pathol 1993;45:345-349.

36. Yamada K, Fukushima T. Mechanisms of cytotoxicity of paraquat: II. Organ specificity of paraquat-stimulated lipid peroxidation in the inner membrane of mitochondria. Exp Toxicol Pathol 1993;45:375-380.

37. Fukushima T, Gao T, Tawara T, Hojo N, Isobe A, Yamane $Y$. Inhibitory effect of nicotinamide to paraquat toxicity and the reaction site on complex I. Arch Toxicol 1997;71:633-637.

38. Imlay JA, Linn S. DNA Damage and Oxygen Radical Toxicity. Science 1988;240:1302-1309.

39. Boveris A, Cadenas E. Mitochondrial production of superoxide anions and its relationship to the antimycin insensitive respiration. FEBS Lett 1975;54:311-314.

40. Boveris A, Cadenas EA, Stoppani O. Role of ubiquinone in the mitochondrial generation of hydrogen peroxide. Biochem J 1976;156:435-444.

41. Boveris A, Chance B. The mitochondrial generation of hydrogen peroxide. General properties and effect of hyperbaric oxygen. Biochem J 1973;134:707-716.

42. Kowaltowski AJ, Castilho RF, Vercesi AE. Opening of the mitochondrial permeability transition pore by uncoupling or inorganic phosphatase in the presence of $\mathrm{Ca}^{2+}$ is dependent on mitochondrial-generated reactive oxygen species. FEBS Lett 1996;378:150-152.

43. Kowaltowski AJ, Costa ADT, Vercesi AE. Activation of the uncoupling mitochondrial protein inhibits reactive oxygen species generation by the respiratory chain. FEBS Lett 1998;425:213-216.

44. Hart JJ, DiTomaso JM, Linscott DL, Kochian LV. Transport interactions between paraquat and polyamines in roots of intact maize seedlings. Plant Physiol 1992;99:1400-1405.

45. Han D, Williams E, Cadenas E. Mitochondrial respiratory chain-dependent generation of superoxide anion and its release into the intermembrane space. Biochem J 2001;353:411-416.

46. Butler J, Koppenol W, Margoliash E. Kinetics and mechanism of the reduction of ferricytochrome $\mathrm{c}$ by the superoxide anion. J Biol Chem 1982;257:10747-10750.

47. Bus JS, Aust SD, Gibson JE. Lipid peroxidation: A possible mechanism for paraquat toxicity. Res Commun Chem Pathol Pharmacol 1975;11:31-38.

48. Leonardis SD, Lorenzo GD, Borraccino G, Dipierro S. A specific ascorbate free radical reductase isozyme participates in the regeneration of ascorbate for scavenging toxic oxygen species in potato tuber mitochondria. Plant Physiol 1995;109:847-851. 